\title{
Trop-2 targeted CAR-T cells inflict enhanced killing of ovarian cancer cells
}

\author{
Yaru Xu ${ }^{1,2}$,Qi Tang ${ }^{2}$,Xiaochen Huang ${ }^{1,2}$,Tingting Yang ${ }^{1,2}$,Wei Zhao ${ }^{1,2}$,Xingwang Kuai ${ }^{1,2}, J_{i n}$ Zhu ${ }^{2,3^{*}}$ \\ ${ }^{1}$ Department of Pathology, Nanjing Medical University, Nanjing, Jiangsu, 211166, China; \\ ${ }^{2}$ Key Laboratory of Antibody Technique of Ministry of Health, Nanjing Medical University, Nanjing, Jiangsu, 211166, China; \\ ${ }^{3}$ Huadong Medical Institute of Biotechniques, Nanjing, Jiangsu, 210002, China
}

\begin{abstract}
$\mathrm{T}$ cell modified by chimeric antigen receptor (CAR) is considered one of the most promising tumor therapies for its almost magic effect in the treatment of hematologic malignancies. Encouraged by breakthroughs in this area, we produced Trop-2-redirected chimeric antigen receptor-modified T (Trop-2 CAR-T) cells and tested their function on ovarian cancer cells with in vitro molecular cloning. Gene recombination technology was introduced to construct the Trop-2 CAR vector. The expression of Trop-2 CAR on 293T cells was tested by Western blot. The effect of Trop-2 CAR-T cells on the proliferation of ovarian cancer cells was evaluated by CCK-8 assay. We found that Trop-2 CAR-T cells prominently inhibited the growth of ovarian cancer cells with Trop-2 antigen expression $(P<0.05)$, moreover, high levels of IFN $-\gamma$ and IL-2 were detected in supernatant by ELISA $(P<0.01)$. These results suggest that Trop-2 CAR-T cells have the potential to be a clinical treatment for patients with Trop-2 positive ovarian cancer.
\end{abstract}

Keywords: Trop-2, chimeric antigen receptor, ovarian cancer

\section{INTRODUCTION}

Owing to the lack of typical clinical symptoms, $60 \%$ to $80 \%$ of all ovarian cancers are diagnosed at an advanced stage, and is the most common cause of mortality due to gynecological malignancy in women; with a 5 -year overall survival rate of less than $30 \%$. All these indicate that ovarian cancer is a very serious threat to the female health ${ }^{[1,2]}$. Currently, the primary treatment protocol for ovarian cancer is the combination of surgery and chemotherapy. Unfortunately, after initial treatment the majority of patients relapse and become chemotherapy-tolerant or chemotherapy-resistant ${ }^{[3,4]}$. Therefore, a new method of treatment is needed.

In recent years, chimeric antigen receptor $\mathrm{T}$ cell
(CAR-T) immunotherapy has experienced a rapid development. The $\mathrm{T}$ cells modified by CAR are endowed with abilities to identify and kill tumor cells specifically. Nowadays, CAR-T immunotherapy is widely used in studies in the targeted therapy of tumors ${ }^{[5]}$. Presently, CAR-T technology has made breakthroughs in the treatment of hematological malignancies ${ }^{[6-8]}$, but there are few reports on the treatment of solid tumors, and reports on the treatment of ovarian cancer in human are rare ${ }^{[9-11]}$.

Human trophoblast cell surface antigen 2 (Trop-2) is a single-transmembrane cell-surface glycoprotein encoded by tumor associated calcium signal transducer 2 (TACSTD2) gene. It serves as a cell surface receptor and plays a role in transducing calcium signals ${ }^{[12,13]}$. Trop- 2 is involved in the regulation of cell adhesion.

*Correspondence to: Jin Zhu, Huadong Medical Institute of Biotechniques, Nanjing, Jiangsu, 210002, China. Tel:+86-25-86869411, Fax: +86-2586869411, E-mails: zhujin1968@njmu.edu.cn.

The authors reported no conflict of interests. 
Through the pathway of cyclin Dl and extracellular regulated protein kinase, it can also promote the proliferation, invasion and metastasis of tumors by upregulating the expression of some downstream signalling molecules which can regulate tumor growth, such as STAT1, STAT3, NF- $\kappa$ B, Rb, and so on ${ }^{[6-7,13]}$. Treropola et al. analyzed the expression of Trop-2 in different ovarian cancer tissues by immunohistochemistry. The results showed that the expression rate of Trop-2 reached $66 \%$ and Trop-2 was over-expressed in $38 \%$ of ovarian cancers ${ }^{[14]}$. Bignotti et al. demonstrated that humanized anti-Trop-2 antibody hRS7 could produce antibody-mediated cytotoxicity in poorly differentiated intrauterine-like adenocarcinoma cells with overexpressed Trop- $2^{[15]}$. The humanized anti-Trop-2 Fab fragment prepared by Lin et al. could induce the apoptosis of breast cancer cells and inhibit the proliferation, invasion and metastasis of breast cancer cells ${ }^{[16]}$. There is no doubt that Trop-2 is a potential target in the targeted therapy of ovarian cancer ${ }^{[8,17]}$. Previously, we had screened out a humanized antibody which had a high affinity for Trop- $2^{[18]}$. In our current study, we produced Trop-2 CAR-T cells based on the Fab fragment of the anti-Trop-2 antibody. We tested their effects on ovarian cancer cells expressing Trop-2 in vitro and preliminarily explored their potential mechanism of action. Our study provided a novel approach for targeted therapy of ovarian cancer.

\section{MATERIALS AND METHODS}

\section{Cells and reagents}

Human ovarian cancer cell lines (OVCAR-3, SKOV3, HO8910, A2780), human breast cancer cell line $(\mathrm{MCF}-7)$, human melanoma cell line (A375), 293T cells, genetically modified retrovirus vector, CD19 CAR vector and packaging plasmids (RD114, peqpam3) were provided by Key Laboratory of Antibody Technique of Ministry of Health affiliated to NJMU. E.coli DH5 a and In-Fusion ${ }^{\circledR}$ HD Cloning Kit were purchased from Clontech Company (USA). Restriction endonucleases (Nco I and $B a m \mathrm{H}$ I ) were purchased from NEB Company (USA). Anti-human Trop-2 (EGP-1) PE, anti-human CD28 antibody, anti-human CD3 antibody, human IFN $-\gamma$ Ready-SET-Go ELISA kit and human IL-2 Ready-SET-Go ELISA kit were purchased from eBioscience Company (USA). Mouse anti-human CD3 $\zeta$ antibody and lymphocyte separation medium were purchased from Stem Cell Company (USA). $100 \mathrm{kDa}$ centrifugal filter device were purchased from Millipore Company (USA). 293fectin ${ }^{\text {TM }}$ Transfection Reagent was purchased from Invitrogen Company (USA). Cell counting kit was purchased from Dojindo Company (Japan).

\section{Construction of recombinant retrovirus vector expressing Trop-2 CAR}

The Trop-2 CAR vector contains genetic elements coding anti-Trop-2 scFv and retroviral expression cassette (coding human IgG CH2CH3 and CD28-CD137CD3 $\zeta$ domain). The primers of light and heavy chains of anti-Trop-2 Fab were designed according to the principle of In-Fusion PCR. The light and heavy chains were amplified and connected with Linker (Gly4Ser)3 vector by overlap PCR to produce a recombinant gene fragment anti-Trop-2 scFv. Anti-Trop-2 scFv was connected with linearized retrovirus vector digested by restriction endonucleases ( $\mathrm{NcO}$ I and $\mathrm{BamH}$ I ) to construct Trop-2 CAR vector. The Trop-2 CAR vector was transferred into E.coli DH5 a. Some positive clones were picked out and analyzed by sequencing technology. The right vector was named Trop-2 CAR.

\section{Virus packaging, expression and identification of the Trop-2 CAR}

The Trop-2 CAR or CD19 CAR was co-transfected with RD114 and peqpam3 into $293 \mathrm{~T}$ cells respectively by $293 \mathrm{fectin}^{\mathrm{TM}}$ Transfection Reagent to produce retrovirus stock. Trop-2 CAR and CD19 CAR retrovirus supernatant were collected at $48^{\text {th }} \mathrm{h}$ and $72^{\text {th }} \mathrm{h}$ after transfection and concentrated by $100 \mathrm{kDa}$ centrifugal filter device. After aseptic treatment, the retrovirus supernatant was stored at $-80^{\circ} \mathrm{C}$.

We chose CD3 $\zeta$ as the target to test the expression of Trop-2 CAR protein on eukaryotic cells by Western blot. 293T cells infected by Trop-2 CAR retrovirus supernatant or not were lysed by RIPA buffer. The samples were denatured and fractionated by $10 \%$ SDSPAGE. Then the samples were blotted onto PVDF membrane and incubated by mouse anti-human CD3 $\zeta$ antibody $(1: 1,000)$ followed by goat anti-mouse $\operatorname{IgG}$ $(1: 5,000)$. The blot was detected by the ECL Western blot analysis system.

\section{Preparation of Trop-2-targeted CAR-T cells}

Peripheral blood mononuclear cells (PBMCs) were separated from $10 \mathrm{~mL}$ peripheral blood from healthy volunteers using lymphocyte separation medium. PBMCs were activated in 24-well plates coated with antihuman CD28 and anti-human CD3 antibodies for 24 hours. From the third day, IL-2 (200 U/mL) was added into medium (RPMI1640+10\%FBS) to promote the proliferation of $\mathrm{T}$ cells. Retrovirus supernatant was 
added to retronectin-coated no-tissue plates for $1 \mathrm{hr}$ and then activated $\mathrm{T}$ cells were seeded into plates to produce $2 \times 10^{8}$ Trop-2-targeted CAR-T (Trop-2 CAR-T) cells. CD19-targeted CAR-T (CD19 CAR$\mathrm{T}$ ) cells (used as control) were prepared in the same method.

\section{The effects of Trop-2 CAR-T cells on ovarian cancer cells in vitro}

Effector cells (Trop-2 CAR-T, CD19 CAR-T and activated T cells) and target cells (HO8910, A2780) were collected in logarithmic growth phase and diluted using medium (RPMI1640 + 10\% FBS). $1 \times 10^{6}$ effector cells were mixed with $100 \mu \mathrm{L}$ of double ratio diluted target cells (at a concentration of $5 \times 10^{5}, 2 \times 10^{5}$, $1 \times 10^{5}, 5 \times 10^{4}$ cells $/ \mathrm{mL}$ ) and co-incubated in wells of 96 -well plates at $37^{\circ} \mathrm{C}$ for 12 hours. The Plates were centrifuged at 1,500 rpm for 5 minutes and the supernatant was discarded. $10 \mu \mathrm{L}$ of CCK-8 was added into each well and incubated with cells at $37^{\circ} \mathrm{C}$ for 3 hours. The absorbance at $450 \mathrm{~nm}$ was measured by multiskan spectrum system (Thermo Labsystems, USA).

CCK-8 assay was also introduced to identify the cytotoxic activity of Trop-2 CAR-T cells against tumor cells (OVCAR-3, HO8910, A2780, SKOV3, MCF-7) expressing a distinct level of Trop-2 at a 20:1 ratio of the effector: target in the same way as previously described. In our cytotoxic assays, CD19 CAR-T cells which shared the same intracellular region with Trop-2 CAR-T cells and activated $\mathrm{T}$ cells, were used as controls.

\section{Cytokine ELISA}

ELISA assay was used to identify the changes of cytokines secreted by Trop-2 CAR-T cells. Antibodies of IFN $-\gamma$ or IL-2 diluted by coating buffer were added into 96-well plates and incubated at $4{ }^{\circ} \mathrm{C}$ for one night. The next day, blocking reagent was added into wells and incubated for 1 hour at $37{ }^{\circ} \mathrm{C}$. The coculture supernatantat was set at a 20:1 ratio of effector : target was collected and added into 96-well plates at $4^{\circ} \mathrm{C}$ overnight. The assay was performed in triplicate. The next day, the biotin-labeled detection antibody was added into plates and incubated for 1 hour following by incubating with HRP-labeled avidin for 30 minutes. At last, TMB buffer was used as developer and the absorbance at $450 \mathrm{~nm}$ was measured by a microplate reader.

\section{Statistical analysis}

The experimental data were analyzed by SPSS 20.0 software package. Measurement data were presented in the form of mean \pm standard deviation $(\bar{x} \pm s)$. ANOVA was used to calculate the significance of difference among multiple groups. Student's $t$ test was used to evaluate the difference between two treatment groups. The difference was considered statistically significant when the $P$ value was less than 0.05 .

\section{RESULTS}

\section{Construction of Trop-2 CAR}

The $V_{k}$ gene and $V_{H}$ gene were amplified based on anti-Trop-2 Fab by PCR at a length of 350 bp and 400 bp (Fig. 1A). Anti-Trop-2 scFv was constructed by overlap PCR at a length of 750 bp (Fig. 1B, lane3). The results of $1 \%$ gel electrophoresis were in line with expectations. Anti-Trop-2 scFv was connected with linearized retrovirus vector to produce Trop-2 CAR. The sequencing results of positive clones transformed with Trop-2 CAR were in accordance with previous design.

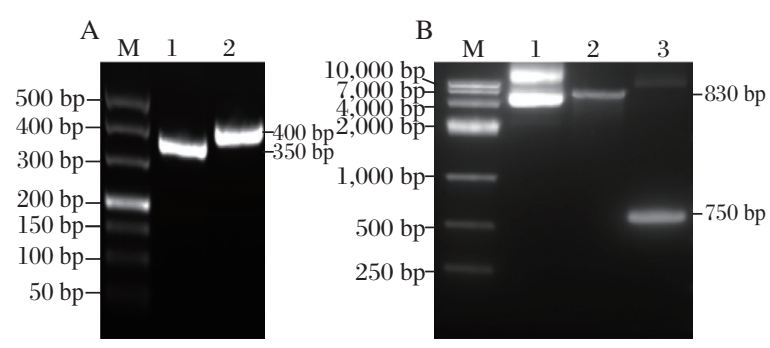

Fig.1 Construction and identification of retrovirus vector expressing Trop-2 chimeric antigen receptor. A: Productions of anti-Trop-2 $\mathrm{V}_{\mathrm{k}}$ and anti-Trop-2 $\mathrm{V}_{\mathrm{H}}$. M: DL500 DNA Marker; 1 : $\mathrm{V}_{\mathrm{k}} ; 2$ : $\mathrm{V}_{\mathrm{H}}$. B: Identification of components of Trop-2 CAR. M: DL10000 DNA Marker; 1: Fragments consisted by $\mathrm{V}_{\mathrm{k}}, \mathrm{V}_{\mathrm{H}}$, (Gly4Ser)3 and linearized retrovirus vector; 2: Linearized retrovirus vector digested by Nco I and BamH I ; 3. Fragments consisted by $\mathrm{V}_{\mathrm{k}}, \mathrm{V}_{\mathrm{H}}$ and (Gly4Ser)3.

\section{Virus packaging, expression and identification of the Trop-2 CAR}

Western blot was used to identify the expression of Trop-2 CAR on 293T cells. CD3 $\zeta$ domain was selected as target. The size of endogenic $\mathrm{CD} 3 \zeta$ protein in T cells was $22 \mathrm{kDa}$, while the size of CD3 $\zeta$ protein in CAR was $75 \mathrm{kDa}$. The results suggested that lanes of 293 T cells transfected with Trop-2 CAR or CD19 CAR had bands of $75 \mathrm{kDa}$, but there was no same band in 293T cells without transfection (Fig. 2).

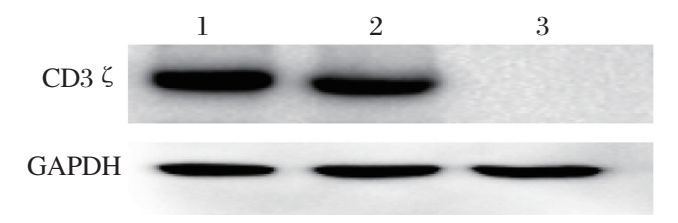

Fig.2 Expression and identification of the Trop-2 CAR on 293T cells. 1: 293T cells transfected with Trop-2 CAR; 2: 293T cells transfected with CD19 CAR; 3: 293T cells without transfection. 


\section{Cytotoxicity of Trop-2 CAR-T cells against ovarian cancer cells in vitro}

CCK-8 assay was used to assess the functionality of Trop-2 CAR-T cells. We chose Trop-2 positive (HO8910) and Trop-2 negative (A2780) ovarian cancer cells as target cells. The killing efficiencies of Trop-2 CAR-T cells against HO8910 were (74.96 \pm 7.24$) \%$, $(48.71 \pm 2.47) \%,(30.93 \pm 5.40) \%$ and $(16.12 \pm 5.21) \%$ respectively at the effector:target ratio of 20:1, 10:1, 5:1, 2:1. Neither CD19 CAR-T cells nor non-transfected T cells killed HO8910 effectively. The killing efficiencies of Trop-2 CAR-T cells against HO8910 were significantly higher than CD19 CAR-T cells or nontransfected T cells $(P<0.05$, Fig. $3 \boldsymbol{A})$. The difference had no statistical significance among various $\mathrm{T}$ cells against A2780 $(P>0.05$, Fig. 3B).
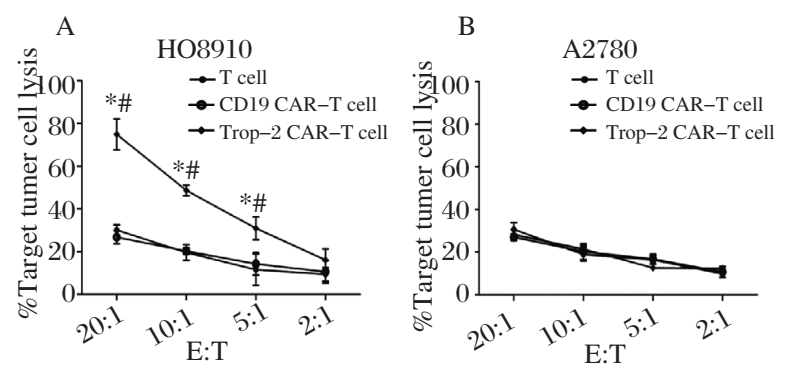

Fig. 3 CCK-8 analysis of the cytotoxic activity of Trop2 CAR-T cells to target cells. A: The cytotoxic activity of Trop-2 CAR-T cells against Trop-2 positive HO8910 at different effector:target ratio. B: The cytotoxic activity of Trop-2 CAR-T cells against Trop2 negative A2780 at different effector:target ratio. Trop-2 CAR-T cell group compared with $\mathrm{T}$ cell group, ${ }^{*} P<0.05$; Trop-2 CAR-T group compared with CD19 CAR-T cell group, ${ }^{\sharp} P<0.05$.

Trop-2 was over-expressed in tumor cells OVCAR-3, HO8910, MCF-7 and expressed at a low level in SKOV3, but not expressed in A2780 or melanoma cells A375 $5^{[18-20]}$. We tested the cytotoxicity of different effector cells against these tumor cells at 20:1 ratio of effector:target in the same method described above(Fig. 4). The killing efficiencies of Trop-2 CAR-T cells against Trop-2 positive tumor cells OVCAR-3, HO8910, SKOV 3 and MCF-7 were $(83.83 \pm 1.23) \%,(72.73 \pm 1.34) \%,(51.63 \pm 3.67) \%$ and $(78.3 \pm 4.45) \%$, respectively. The killing efficiencies were significantly higher than the CD19 CAR$\mathrm{T}$ cell group or non-transfected $\mathrm{T}$ cell group $(P<$ $0.05)$. There was no statistical difference between the CD19 CAR-T cell group and non-transfected T cell group $(P>0.05)$. The killing efficiencies of Trop2 CAR-T cells against Trop-2 negative tumor cells A2780 and A375 were respectively $(23.53 \pm 1.10) \%$ and $(21.23 \pm 1.36) \%$. The difference had no statistical significance among different groups $(P>0.05)$.

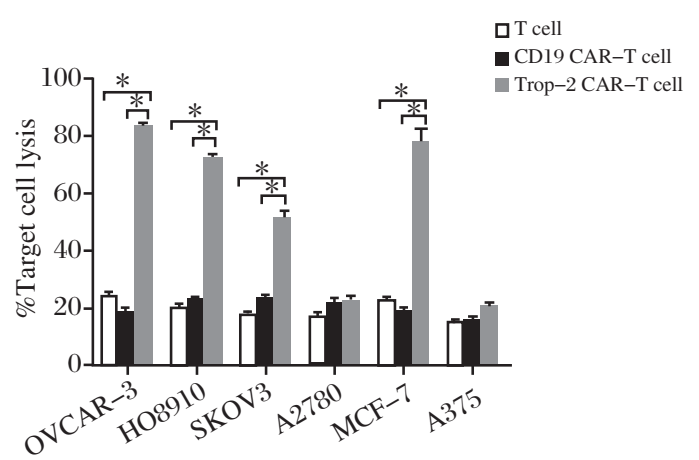

Fig.4 CCK-8 analysis of the cytotoxic activity of Trop-2 CAR-T cells with different Trop- 2 expression. ${ }^{*} P<0.05$.

\section{Trop-2 CAR-T cells produce IFN- $\gamma$ or IL-2 in a Trop-2 specific manner}

Trop-2 CAR-T cells were co-incubated with ovarian cancer cells (OVCAR-3, HO8910, SKOV3, A2780) for $48 \mathrm{hrs}$ and IFN- $\gamma$ or IL-2 in their culture supernatant were tested by ELISA (Fig. 5). When Trop-2 CAR-T cells were co-cultured with Trop2 positive ovarian cancer cells (OVCAR-3, HO8910, SKOV3), the content of IFN $-\gamma$ in supernatant was $(1,813 \pm 100.5) \mathrm{pg} / \mathrm{mL},(1,655.2 \pm 22.3) \mathrm{pg} / \mathrm{mL}$ and $(1,404.7 \pm 68.98) \mathrm{pg} / \mathrm{mL}$, respectively. The content of IL-2 was $(1,707.9 \pm 113.3) \mathrm{pg} / \mathrm{mL},(1,511.2 \pm 131.3)$ $\mathrm{pg} / \mathrm{mL}$ and $(842 \pm 173.45) \mathrm{pg} / \mathrm{mL}$. The content of IFN $-\gamma$ and IL-2 secreted by Trop-2 CAR-T cells was higher than CD19 CAR-T cell group and T cell $\operatorname{group}(P<0.01)$. There was no statistical significance between the CD19 CAR-T cell group and the nontransfected $\mathrm{T}$ cell group $(P>0.05)$. When Trop-2 CAR-T cells were co-cultured with Trop-2 negative ovarian cancer cells A2780, the content of IFN $-\gamma$ in supernatant was $(234.23 \pm 14.8) \mathrm{pg} / \mathrm{mL}$. The content of IL-2 was $(31.7 \pm 3.4) \mathrm{pg} / \mathrm{mL}$. There was no statistical significance among different groups $(P>0.05)$.

\section{DISCUSSION}

Since Gross et al. proposed the concept of CAR in 1989, CAR-T technology has been improved continuously. In recent years, CAR-T technology has made remarkable breakthroughs in the treatment of hematological malignancies ${ }^{[6-8]}$. These studies initially confirmed the potential of CAR-T cells to cure tumors. Then more researchers devoted into studies on the treatment of solid tumors by CAR-T technology ${ }^{[9,21,22]}$. At present, the lack of tumor specific antigen is a main limiting factor for the therapeutic effects of CAR-T technology in the field of solid tumors ${ }^{[23]}$. As mentioned above, our early experiments and other researches suggested that Trop-2 was over-expressed in ovarian can- 

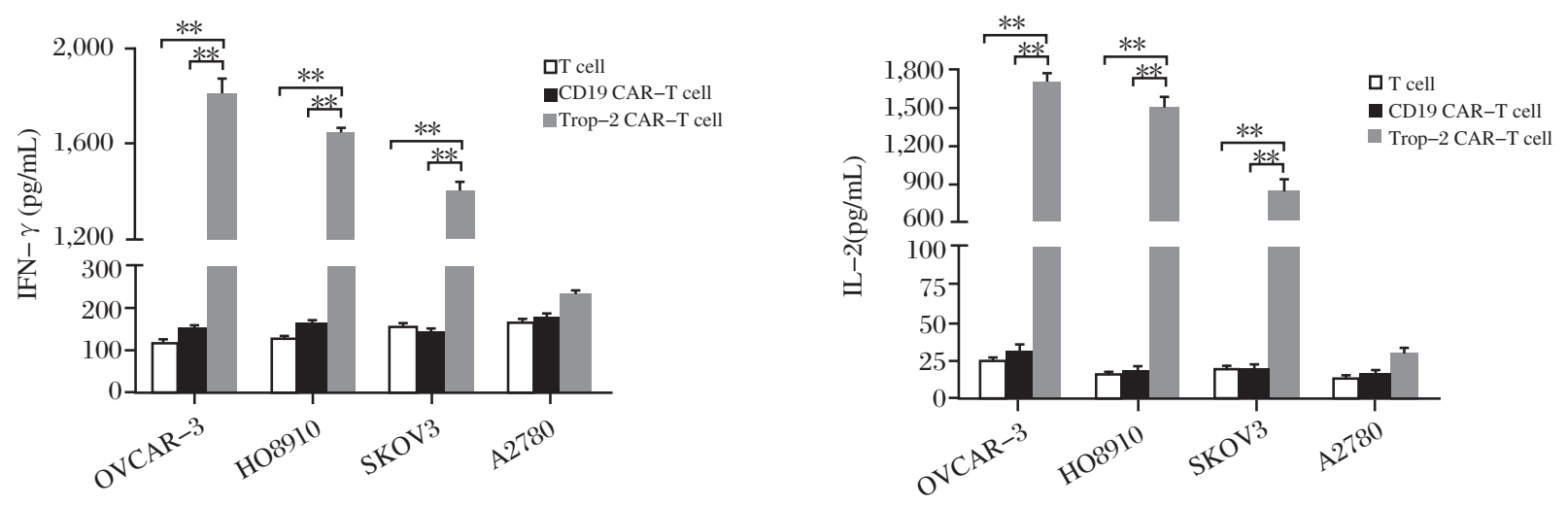

Fig.5 ELISA detection of Trop-2 CAR-T cells secreting IFN- $\gamma(\mathbf{A})$ and IL-2(B). ${ }^{* *} P<0.01$.

cer, but was not expressed or expressed at a low level in normal tissues, moreover its expression was related to aggressive tumor behavior ${ }^{[6,7,13,14,20,24]}$. These studies identified the potential value of Trop-2 as a target in the treatment of ovarian cancer. Previously, a human anti-Trop-2 antibody prepared by our laboratory was verified as having a significant effect on inhibiting the malignant behaviors of ovarian cancer cells expressing Trop-2 protein $^{[18]}$. This study provided evidence of the feasibility for the preparation of Trop-2 CAR-T cells.

We prepared a human extracellular scFv domain of the CAR based on the anti-Trop-2 Fab which has a high affinity for Trop-2. The scFv domain was served as detector of Trop- 2 antigen on tumor cells. The human extracellular domain could prevent patients from human antimouse antibody (HAMA) response after the infusion of CAR-T cells. In our study, intracellular costimulatory signal transduction regions, CD28, CD137 and $\mathrm{CD} 3 \zeta$, were used to improve the survival time and cytotoxic effect of $\mathrm{T}$ cells by enhancing the proliferation ability of T cells in vivo and promoting the release of cytokines. CD19 is the most common target for CAR-T immunotherapy and its value in the treatment of hematological malignancies had been fully validated $^{[6-8]}$. Besides, CD19 is only expressed in normal mature B cells, malignant B cells and precursor B cells, but not expressed in ovarian cancer cells ${ }^{[25,26]}$. For these characters, CD19 CAR (owing the same intracellular domain with Trop-2 CAR) was selected as negative control. CCK-8 assay suggested that the killing effect of Trop-2 CAR-T cells increased along with the increase of effect:target ratio. The killing efficiencies of Trop-2 CAR-T cells against Trop-2 overexpressing ovarian cancer cells HO8910 and OVCAR-3 were more than $70 \%$ and more than $50 \%$ against Trop-2 low-expressing SKOV3. The killing efficiency was significantly improved compared with the CD19 CAR$\mathrm{T}$ cell group and $\mathrm{T}$ cell group. For tumor cells with noexpression of Trop-2, there was no significant differ- ence in the killing efficiency among different groups. The results indicated that Trop-2 CAR-T cells could specifically recognize the Trop- 2 antigen on the surface of the target cells and their cytotoxicity was related to the level of the Trop-2 antigen expressed on the target cell surface. ELISA assay suggested that Trop-2 CAR-T cells secrete more IFN- $\gamma$ and IL-2 when they were co-incubated with Trop-2 positive ovarian cancer cells, compared with the CD19 CAR-T cell group and $\mathrm{T}$ cell group. The co-culture with Trop-2 negative ovarian cancer cells did not induce higher IFN $-\gamma$ and IL-2 release. This indicates that the intracellular signal transduction domain can be activated efficiently after the binding of Trop-2 CAR-T cells with Trop-2 antigen and then improves the cytotoxicity against Trop-2 positive tumor cells by promoting the cytokine secretion of IFN- $\gamma$ and IL-2.

In our study, the 3rd generation Trop-2 CAR-T cells were prepared and were able to kill Trop-2 positive ovarian cancer cells efficiently in vitro. Their killing effects were related to the increased cytokine secretion of IFN $-\gamma$ and IL-2. Our study laid a foundation for the further application of Trop-2 CAR-T cells in the clinical treatment of ovarian cancer.

\section{Acknowledgments and funding}

This work was supported by grants from National Natural Science Foundation of China (NO.81602119).

\section{References}

[1] Yeung TL, Leung CS, Yip KP, et al. Cellular and molecular processes in ovarian cancer metastasis. A review in the theme: cell and molecular processes in cancer metastasis. Am J Physiol Cell Physiol, 2015, 309(7): C444-56.

[2] Jeon SY, Hwang KA, Choi KC. Effect of steroid hormones, estrogen and progesterone, on epithelial mesenchymal transition in ovarian cancer development. J Steroid Biochem Mol Biol, 2016, 158: 1-8.

[3] Hansen JM, Coleman RL, Sood AK. Targeting the tumour microenvironment in ovarian cancer. Eur J Cancer, 
2016,56: 131-43.

[4] Aravantinos G, Pectasides D. Bevacizumab in combination with chemotherapy for thetreatment of advanced ovarian cancer: a systematic review. J Ovarian Res, 2014,7: 57.

[5] Kloss CC, Condomines M, Cartellieri M, et al. Combinatorial antigen recognition with balanced signaling promotes selective tumor eradication by engineered $\mathrm{T}$ cells. Nat Biotechnol, 2013, 31(1): 71-5.

[6] Park JH, Geyer MB, Brentjens RJ. CD19-targeted CAR $\mathrm{T}$-cell therapeutics for hematologic malignancie: interpreting clinical outcomes to date. Blood, 2016, 127(26): 3312-20.

[7] Davila ML, Sadelain M. Biology and clinical application of CAR T cells for B cell malignancies. Int J Hematol, 2016, 104(1): 6-17.

[8] Kochenderfer JN, Dudley ME, Carpenter RO, et al. Donor-derived CD19-targeted $\mathrm{T}$ cells cause regression of malignancy persisting after allogeneic hematopoietic stem cell transplantation. Blood, 2013, 122(25):4129-39.

[9] Whildinga LM, Mahera J. CAR T-cell immunotherapy: The path from the by-road to the free way? J Clin Oncol, 2015, 33(6): 540-9.

[10] Tanyi JL, Stashwick C, Plesa G, et al. Possible compartmental cytokine release syndrome in a patient with recurrent ovarian cancer after treatment with mesothelintargeted CAR-T Cells. J Immunother, 2017, 40(3): 1047.

[11] Kandalaft LE, Powell DJ Jr, Coukos G. A phase I clinical trial of adoptive transfer of folate receptor-alpha redirected autologous $\mathrm{T}$ cells for recurrent ovarian cancer. $J$ Transl Med, 2012, 10: 157.

[12] Goldenberg DM, Cardillo TM, Govindan SV, et al. Trop2 is a novel target for solid cancer therapy with sacituzumabgovitecan (IMMU-132), an antibody-drug conjugate (ADC). Oncotarget, 2015, 6(26): 22496-512.

[13] Zhao W, Tang Q, Kuai XW, et al. Trop2 as an oncogene in gastric cancer by regulating the PI3K/Akt signaling pathway. Asia-Pacific Journal of Blood Types and Genes, 2017, 1(2):21-9.

[14] Trerotola M, Cantanelli P, Guerra E, et al. Upregulation of Trop-2 quantitatively stimulates human cancer growth. Oncogene, 2013, 32(2): 222-33.

[15] Bignotti E, Ravaggi A, Romani C, et al. Trop-2 overexpression in poorly differentiated endometrial endometrioid adenocarcinoma: Implications for immunotherapy with hRS7, a humanized anti-Trop-2 monoclonal antibody.
Int J Gynecol Cancer, 2011, 21(9): 1613-21.

[16] Lin H, Zhang H, Wang J, et al. A novel human Fab antibody for Trop2 inhibits breast cancer growth in vitro and in vivo. Int J cancer, 2014, 134(5):1239-49.

[17] Cardillo TM, Govindan SV, Sharkey RM, et al. Humanized anti-Trop-2 IgG-SN-38 conjugate for effective treatment of diverse epithelial cancers: preclinical studies in human cancer xenograft models and monkeys. Clin Cancer Res, 2011, 17(10):3157-69.

[18] Liu JR, Bai LY, Tang Q, et al. The preparation of human anti-Trop-2 IgG and their effectes on ovarian cancer cells. JBR, 2016, 36(3): 280-6.

[19] Guerra E, Trerotola M, Aloisi AL, et al. The Trop-2 signalling network in cancer growth. Oncogene, 2013, 32(12):1594-600.

[20] Xu N, Zhang Z, Zhu J, et al. Overexpression of trophoblast cell surface antigen 2 as an independent marker for a poor prognosis and as a potential therapeutic target in epithelial ovarian carcinoma. Int J Exp Pathol, 2016,97(2):150-8.

[21] Brown CE, Alizadeh D, Starr R, et al. Regression of glioblastoma after chimeric antigen receptor T-cell therapy. $N$ Engl J Med, 2016, 375(26): 2561-9.

[22] Tang XJ, Zhou Y, Li WJ, et al. T cells expressing a LMP1-specific chimeric antigen receptor mediate antitumor effects against LMP1-positive nasopharyngeal carcinoma cells in vitro and in vivo. JBR, 2014, 28(6): 468-75.

[23] Sadelain M, Brentjens R, Riviere I. The basic principles of chimeric antigen receptor Design. Cancer Discov, 2013,3(4): 388-98.

[24] Trerotola M, Ganguly KK, Fazli L, et al. Erratum: Trop2 is up-regulated in invasive prostate cancer and displaces FAK from focal contacts. Oncotarget, 2015, 6(16): 14318-28.

[25] Chmielewski M, Kopecky C, Hombach AA, et al. IL-12 release by engineered $\mathrm{T}$ cells expressing chimeric antigen receptors can effectively muster an antigen-independent macrophage response on tumor cells that have shut down tumor antigen expression. Cancer Res, 2011, 71(17): 5697-706.

[26] Kochenderfer JN, Yu ZY, Frasheri D, et al. Adoptive transfer of syngeneic $\mathrm{T}$ cells transduced with a chimeric antigen receptor that recognizes murine CD19 can eradicate lymphoma and normal B cells. Blood, 2010, 116(19): 3875-86.

(Received 09 August 2017, Revised 16 August 2017, Accepted 22 August 2017) 